\title{
Graphene: A Rising Star on the Horizon of Materials Science
}

\author{
Ujjal Kumar Sur \\ Department of Chemistry, Behala College, University of Calcutta, Kolkata 700060, India \\ Correspondence should be addressed to Ujjal Kumar Sur, uksur99@yahoo.co.in
}

Received 22 June 2012; Accepted 23 August 2012

Academic Editor: Balaprasad Ankamwar

Copyright ( 2012 Ujjal Kumar Sur. This is an open access article distributed under the Creative Commons Attribution License, which permits unrestricted use, distribution, and reproduction in any medium, provided the original work is properly cited.

Graphene, a one-atom thick planar sheet of $\mathrm{sp}^{2}$ bonded carbon atoms packed in a honeycomb lattice, is considered to be the mother of all graphitic materials like fullerenes, carbon nanotubes, and graphite. Graphene has created tremendous interest to both physicists and chemists due to its various fascinating properties, both observed and predicted with possible potential applications in nanoelectronics, supercapacitors, solar cells, batteries, flexible displays, hydrogen storage, and sensors. In this paper, a brief overview on various aspects of graphene such as synthesis, functionalization, self-assembly, and some of its amazing properties along with its various applications ranging from sensors to energy storage devices had been illustrated.

\section{Introduction}

Since the discovery of fullerene $\left(\mathrm{C}_{60}\right)$ in 1985 by Kroto and his coworkers [1], several novel carbon nanomaterials have been isolated. In 1991, carbon nanotube (CNT) was discovered by Iijima [2], while graphene, a two-dimensional form of graphite, was isolated in 2004 by Novoselov et al. of Manchester University, UK [3]. Graphene has become a new supermaterial due to its unique physical properties. This new kind of two-dimensional carbon nanostructure has attracted tremendous attention both from fundamental and applied research point of view in physics and chemistry as well as materials science. Today, graphene is the most attractive nanomaterial not only because it is the thinnest known material in the universe and the strongest ever measured but also due to its excellent electrical, thermal, mechanical, electronic, and optical properties. It has high specific surface area, high chemical stability, high optical transmittance, high elasticity, high porosity, biocompatibility, tunable band gap, and ease of chemical functionalization which actually helps in tuning its properties [4, 5]. Graphene exhibits many exciting properties such as half-integer room-temperature quantum Hall effect [6], long-range ballistic transport with almost ten times greater electron mobility than of silicon (Si), availability of charge carriers that behave as massless relativistic quasi particle (Dirac fermions), and quantum confinement giving rise to finite band gap and Coulomb blockade effect. Table 1 illustrates some of the amazing physical properties of graphene. These extraordinary properties of graphene can be utilized for making many novel electronic devices such as field-effect transistor (FET), sensors, and supercapacitors. A noteworthy feature of graphene is that the energy of electrons is linearly dependent on the wave vector near the crossing points in the Brillouin zone. The charge carriers which behave as massless relativistic particles can be explained according to Dirac equation rather than the usual Schrodinger equation. Although graphene is expected to be flat, ripples occur due to thermal fluctuations.

Graphene is a flat single sheet from graphite and has the ideal two-dimensional (2D) structure with a monolayer of carbon atoms packed into a honeycomb crystal plane [7]. Figure 1 shows the two-dimensional honeycomb structure of carbon atoms in graphene along with the highresolution transmission electron microscopic (TEM) image. The TEM image clearly shows the hexagonal lattice with carbon-carbon distance $0.14 \mathrm{~nm}$. Graphene is considered as the fundamental building block for graphitic materials of all other dimensions. It can be wrapped up into zerodimensional (0D) fullerenes, rolled into one-dimensional (1D) nanotubes and stacked into three-dimensional (3D) graphite. Therefore, graphene is called the mother of all graphitic carbon-based nanomaterials. Figure 2 illustrates the schematic diagram demonstrating that graphene is the mother of all graphitic forms. 


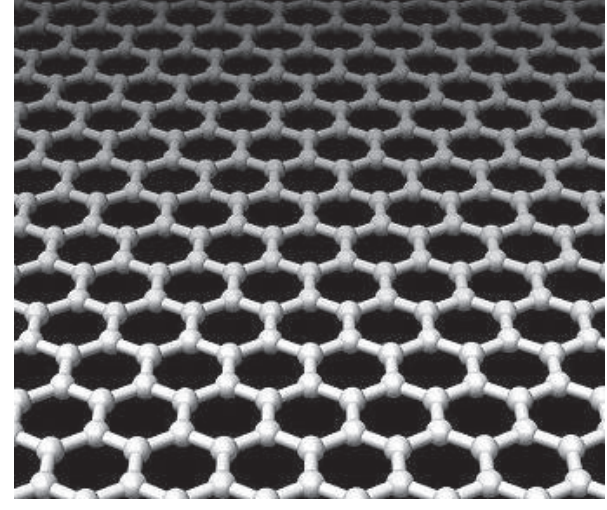

(a)

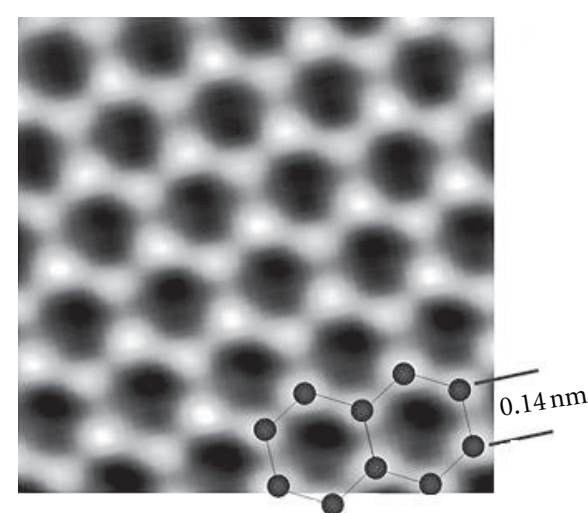

(b)

FIgURE 1: The two-dimensional honeycomb structure of carbon atoms in graphene along with the high-resolution transmission electron microscopic (TEM) image.

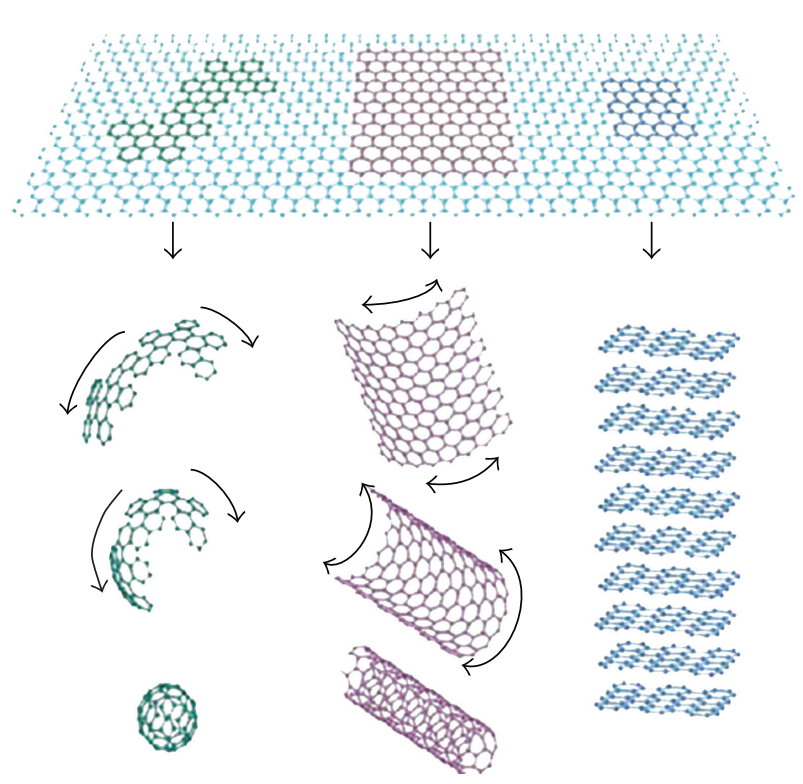

Figure 2: The schematic diagram which show that graphene is the mother of all graphitic form. Reprinted with permission from Geim and Novoselov [4] Copyright (2007) by Macmillan Publishers limited.

Although several review articles had been written on various aspects of graphene, none of these articles covers wide spectrum of synthesis-property-application correlation of graphene. There is also no demonstration of graphene as potential substrate for Raman enhancement in these review articles. In addition to this, the various applications of graphene had not been described in details in most of these review articles.

The aim of this paper is to provide updated overview of graphene in terms of synthesis, historical background, some of the amazing properties, and applications. Due to the space limitations, a complete review of all recent work on this important nanomaterial is not possible. However,
TABLE 1: Some of the amazing properties of graphene.

\begin{tabular}{lc}
\hline Some basic properties & Graphene \\
\hline Young's modulus & $\sim 1100 \mathrm{GPa}$ \\
Fracture strength & $125 \mathrm{GPa}$ \\
Thermal Conductivity & $\sim 5000 \mathrm{~W} \mathrm{~m}^{-1} \mathrm{~K}^{-1}$ \\
Mobility of charge carrier & $2 \times 10^{5} \mathrm{~cm}^{2} \mathrm{~V}^{-1} \mathrm{~s}^{-1}$ \\
Specific Surface area & $2630 \mathrm{~m}^{2} \mathrm{~g}^{-1}$ \\
\hline
\end{tabular}

I have only summarized a few representative examples. In this comprehensive paper, special attention has been paid on the potential use of graphene as energy storage devices such as hydrogen storage and supercapacitors. In addition to this, it has also been shown that graphene can be used as a new novel substrate for Raman enhancement.

\section{Historical Background and Isolation of Graphene}

Theoretically, graphene or 2D graphite has been studied for last sixty years [35]. However, graphene was presumed not to exist in free state and was believed to be thermodynamically unstable with respect to the formation of curved structures such as soot, fullerenes, and nanotubes. More than seventy years ago, Peierls and Landau argued that $2 \mathrm{D}$ crystals were thermodynamically unstable and could not exist in nature [36, 37]. Therefore, it was believed that graphene is an integral part of larger $3 \mathrm{D}$ structures, usually grown epitaxially on top of a monocrystals with matching crystal lattices [38]. Without such a 3D base, 2D materials were supposed not to exist, until 2004 when graphene was experimentally discovered accidentally and unexpectedly by Novoselov et al. [3]. Mechanically exfoliated single-layer graphene was developed using a technique called micromechanical cleavage [3]. Typically, a cellophane tape is used to peel off graphene layers from a graphite flake, followed by pressing the tape against a substrate. Upon removing the tape, a single sheet of graphene is obtained. 
This is also called "Scotch tape" or "Peel-off" method. The follow-up experiments confirmed that graphene consists of charge carriers which were indeed massless Dirac fermions. Later, other free-standing 2D atomic crystals such as singlelayer boron nitride was obtained on top of a noncrystalline substrate [39]. In single-layer graphene and boron nitride, the $2 \mathrm{D}$ crystals were found not only to be continuous but to exhibit high crystal quality $[3,39]$. It can be argued that the extracted 2D crystallites are quenched in a metastable state because they are obtained from 3D materials, whereas their small dimension $(\ll 1 \mathrm{~mm})$ and strong interatomic bonds make sure that thermal fluctuations cannot lead to the generation of dislocations or other crystal defects even at high temperatures.

Before discussing on the synthetic aspects and selected properties of graphene, it is important to define 2D crystals. Generally, a single atomic plane is a 2D crystal, whereas more than 100 layers should be considered as a thin film of 3D crystal. Electronic spectra can be used to differentiate between $2 \mathrm{D}$ and $3 \mathrm{D}$ crystals. Only graphene and its bilayer has simple electronic spectra. They are considered to be zero-bandgap semiconductors with one type of electron and hole. For three or more layers, the electronic spectra become increasingly complex in nature with the appearance of several charge carriers and overlapping of valence and conduction bands. It was shown from the electronic spectra that 3D limit can be approached for 10 or more layers. In the absence of quality graphite wafers, most experimental research groups are currently using samples obtained by micromechanical cleavage of bulk graphite. However, there are several problems, which the researchers are facing to obtain high quality graphene. Only highly ordered or oriented pyrolytic graphite (HOPG) is repeatedly pealed off using cellophane tape followed by deposition onto a silicon substrate. However, yields of graphene are very poor and graphene crystallites left on a substrate are extremely rare and hidden in a multilayer of thousands of thick (graphite) flakes, which can be further characterized by atomic force microscope (AFM), optical microscope, and Raman techniques to provide conclusive evidence on the existence of high quality 2D carbon nanomaterials.

\section{Synthetic Aspects of Graphene}

Though in 2004, Novoselov et al. extracted graphene by micromechanical cleavage of highly oriented pyrolytic graphite (Scotch-tape method) in small yield [3], the next seven-to-eight years have witnessed several different methods for the production of graphene. Ritter and Lyding utilized the mechanical exfoliation method to deposit graphene monolayers and bilayers with minimum lateral dimensions of $2-10 \mathrm{~nm}$ onto a hydrogen passivated $\mathrm{Si}(100)$ surface [40]. These earlier works provided opportunities to experimentally investigate the electronic structure of nanosized graphene and formed the foundation to develop graphenebased nanoelectronics. However, the low throughput of the mechanical exfoliation method largely limits its applications for mass production. Therefore, alternative methods involving a high yield of graphene are highly desirable.
The various methods which have been reported in the literature so far to synthesize graphene sheets include mechanical cleavage of graphite [3], unzipping of carbon nanotubes $[9,10]$, chemical exfoliation of graphite [12], solvothermal synthesis [13], epitaxial growth on silicon carbide (SiC) surfaces and metal surfaces $[17,41]$, chemical vapour deposition (CVD) of hydrocarbons on metal surfaces [42], bottom-up organic synthesis [14], and the reduction of graphene oxide obtained from graphite oxide by a variety of reducing agents [18-22]. The last mentioned protocol yields only chemically modified graphene.

On the other hand, single- and few-layer graphene have been grown epitaxially by chemical vapour deposition of hydrocarbons on metal substrates $[16,42]$. Such films were studied by surface science techniques such as AFM and TEM. Only lately, few-layer graphene obtained on SiC was characterized with respect to its electronic properties, revealing high-mobility charge carriers. Single-layer graphene can also be synthesized by epitaxial growth on $\mathrm{SiC}$, in which single crystal $\mathrm{SiC}$ substrates or commercial polycrystalline $\mathrm{SiC}$ granules were heated in vacuum at a high temperature between 1200 to $1600^{\circ} \mathrm{C}$. Due to the higher sublimation of silicon over carbon, excess carbon, which is left behind on the surface, can rearrange to form graphene. Epitaxial growth of graphene provides possibly the only feasible route towards electronic applications.

Large area and high-quality graphene can also be synthesized using CVD technique by decomposition of several hydrocarbons such as methane, ethylene, acetylene, and benzene on substrates like nickel $(\mathrm{Ni})$, copper $(\mathrm{Cu})$, cobalt $(\mathrm{Co})$, and ruthenium $(\mathrm{Ru})$ [16]. A radio frequency plasma enhanced chemical vapour system was used to synthesize graphene on substrates such as $\mathrm{Si}, \mathrm{W}, \mathrm{Mo}, \mathrm{Zr}, \mathrm{Ti}, \mathrm{Cr}$, $\mathrm{SiO}_{2}$, and $\mathrm{Al}_{2} \mathrm{O}_{3}$ [43]. This process reduces the energy consumption and prevents the formation of amorphous carbon and other unwanted byproducts.

The chemical method is considered as a scalable approach to obtain graphene at a large scale. This method has been used widely to synthesize chemically derived graphene. Graphite is first oxidized to graphite oxide using either the Hummers method [44] or the modified Hummers method [45] in the presence of strong acids and oxidants. Graphite oxide can be readily exfoliated as individual graphene oxide $(\mathrm{GO})$ sheets by ultrasonication in water. $\mathrm{GO}$, an oxidized form of grapheme, is decorated by hydroxyl and epoxy functional groups on the hexagonal network of carbon atoms with carboxyl groups at the edges. GO is highly hydrophilic and forms stable aqueous colloids due to presence of large number of oxygen-containing functional groups and electrostatic repulsions [19]. It is electrically insulating and can be converted into conducting graphene by chemical reduction process. GO readily exfoliates upon sonication in water. It is also interesting to note that GO can be directly dispersed in several polar solvents like ethylene glycol, dimethylformamide, $\mathrm{N}$-methylpyrrolidone, and tetrahydrofuran. Chemically derived graphene can be then obtained after reduction of the GO through deoxygenation using hydrazine solution or any other reducing agent. There have been reports in the literature on the reduction of 
GO in solution phases using different reducing agents such as hydrazine, dimethylhydrazine, hydroquinone, ethylene glycol, sodium borohydride, lithium borohydride $[18,23$, $45,46]$, and in the vapour phase using hydrazine/hydrogen or just by thermal annealing [47]. Therefore, GO is an excellent precursor to synthesize graphene nanosheets. It was shown that few-layer graphene can be obtained under mild conditions using ascorbic acid [48]. Few-layer graphene can also be synthesized using sugars such as glucose, fructose, and sucrose as the reducing agents [49]. Microwave [5, 24], laser [25], plasmas [26], sonochemical [27] as well as hydrothermal techniques [28] were also employed to synthesize graphene from GO. In the hydrothermal technique [28], water behaves as supercritical liquid and plays the role of reducing agent. GO can also be reduced photochemically employing ultraviolet (UV) radiation to synthesize graphene using $\mathrm{H}_{3} \mathrm{PW}_{12} \mathrm{O}_{40}$ as a photocatalyst [29]. It has been demonstrated by Salas and his group that graphene has been produced by the reduction of GO using environmental bacteria as an electron donor [50].

Graphene has been produced by heating nanodiamond in an inert atmosphere $[51,52]$. In this procedure, pristine nanodiamond powder (particle size of 4-6 nm) was heated in a graphite furnace in a helium atmosphere at different high temperatures $\left(1650,1850,2050\right.$, and $\left.2200^{\circ} \mathrm{C}\right)$ for one hour. Subrahmanyam and his group had synthesized graphene by arc evaporation of graphite in the presence of hydrogen [53]. This protocol yields graphene sheets with 2-3 layers with flake size of 100-200 nm.

Stankovich's group had demonstrated a solution-based process for the production of chemically derived single-layer graphene with an excellent stability [20]. A two-step method was reported by Gao and his group for nearly complete reduction of surface functionalities of GO by deoxygenation with $\mathrm{NaBH}_{4}$ and dehydration with concentrated sulfuric acid [54]. However, the harsh oxidation and reduction reactions may deteriorate the graphene structure and decrease the performance of graphene-based devices.

Ang et al. proposed a straightforward one-step intercalation and exfoliation method to produce large-sized, conductive graphene sheets with a high yield (>90\%) without the use of surfactants [55]. The method is based on the rich intercalation chemistry of GO aggregates. Graphene can be synthesized by the liquid-phase exfoliation of graphite [15] in organic solvents. Exfoliation of graphite in $\mathrm{N}$ methyl pyrrolidone or surfactant/water solution employing ultrasonication also yields stable single-layer graphene dispersions $[15,56]$.

Kosynkin and his co-workers at the Rice University, Texas, USA synthesized graphene nanoribbons on a large scale by a simple solution-based oxidative longitudinal unzipping of multiwalled carbon nanotubes [9]. They also synthesized pristine graphene from unexpected and incredible starting materials like food, waste, and insect [34]. The protocol used by Ruan and his group involves the synthesis of high-quality graphene monolayer from incredible and less expensive solid carbon materials as a precursors such as cookies and chocolates (food), grass, plastics and dog faeces (waste), and cockroaches (insects) without any purification step [34]. This group has demonstrated the growth of monolayer pristine graphene from solid carbon sources atop a copper foil. To obtain graphene monolayer, $10 \mathrm{mg}$ of the dry carbon source was placed atop the copper foil supported in a quartz boat and annealed in a tubular furnace at $1050^{\circ} \mathrm{C}$ for $15 \mathrm{~min}$ under low pressure $\mathrm{H}_{2}$ and Ar. When grass and dog faeces were employed as carbon precursors for the removal of moisture, the samples were heated in a $65^{\circ} \mathrm{C}$ vacuum (102 torr) oven for 10 hours. It is expected that the protocol used by Tour and his group from less expensive carbon sources will reduce the cost of graphene rapidly in the commercial market and will be extremely popular for largescale synthesis of graphene.

Shinde and his group at the National Chemical laboratory, Pune, India introduced a new protocol for the facile synthesis of high quality of graphene nanoribbons by two-step electrochemical approach [10]. In this protocol, multiwalled carbon nanotubes were transformed into graphene nanoribbons by a process known as "electrochemical unzipping." This process consists of the oxidation of multiwalled carbon nanotubes electrochemically at a controlled potential, followed by reduction either chemically or electrochemically to yield graphene layer with defects or without defects.

A few groups had synthesized graphene under electrochemical conditions [30-32]. Wang et al. [30] synthesized graphene by direct electrochemical reduction of single-layer graphene oxide and subsequently functionalize graphene with glucose oxidase. Highly efficient and largescale protocol was employed [31] to synthesize graphene from graphite by electrolytic exfoliation using poly(sodium4-styrenesulfonate) as an effective electrolyte. Recently, Liu et al. [32] carried out a simple two-step electrochemical technique to produce graphene sheets films on indium-tin oxide (ITO) electrode. The main procedure involves the electrophoretic deposition of graphene oxide film onto ITO electrode followed by the in situ electrochemical reduction of graphene oxide to generate graphene sheet film. Table 2 illustrates the various synthetic protocols employed for the synthesis of graphene.

\section{Some Properties of Graphene}

4.1. Functionalization and Solubilization. Pristine graphene is insoluble in liquids such as water, polymer resins and other common solvents. Therefore, it is necessary to attach certain functional groups on graphene either physically or chemically to disperse in various common solvents without significantly changing its desirable properties. Functionalization of graphene has been carried out by different groups using various novel strategies [57]. Niyogy and coworkers have functionalized graphene by employing covalent attachment [58]. Amidation was carried out to solubilize graphene in nonpolar solvent. In this process, acid-treated graphene containing surface $-\mathrm{OH}$ and $-\mathrm{COOH}$ groups was first treated with thionyl chloride $\left(\mathrm{SOCl}_{2}\right)$, followed by reaction with a long chain aliphatic amines. Soluble graphene layers in tetrahydrofuran (THF) was obtained by the covalent attachment of alkyl chains to graphene layers via reduction 
TABLE 2: Various methods employed for the synthesis of graphene.

\begin{tabular}{|c|c|c|}
\hline Synthetic protocol & Yield & Reference \\
\hline Micromechanical cleavage (Scotch-tape method) & Low & Novoselov et al. [3] \\
\hline Unzipping of carbon nanotubes (mechanical and electrochemical unzipping) & \multicolumn{2}{|c|}{ Moderate Kosynkin et al. [9], Shinde et al. [10 } \\
\hline Solvothermal synthesis & Moderate & Choucair et al. [13] \\
\hline Bottom-up organic synthesis & High & Yang et al. [14] \\
\hline Thermal decomposition of $\mathrm{SiC}$ & High & Emtsev et al. [17] \\
\hline Chemical reduction of graphene oxide obtained from graphite oxide by various reducing agents & High & {$[18-23]$} \\
\hline Microwave, laser, plasmas, sonochemical, hydrothermal, and photochemical techniques & Moderate & [24-29] \\
\hline Electrochemical method & High & {$[30-32]$} \\
\hline
\end{tabular}

of graphite fluoride with alkyl lithium reagents [59]. Such covalent functionalization facilitates the solubilization in various solvents such as $\mathrm{CCl}_{4}, \mathrm{THF}$, and $\mathrm{CH}_{2} \mathrm{Cl}_{2}$. Water soluble graphene can be prepared by the reaction of pristine graphene with a mixture of concentrated $\mathrm{H}_{2} \mathrm{SO}_{4}$ and $\mathrm{HNO}_{3}$. This water-soluble graphene is stable for several months. Si and Samulski carried out a facile and scalable procedure to synthesize aqueous solutions of isolated, sparingly sulfonated graphene [23]. ${ }^{13} \mathrm{C}$ NMR and FTIR spectra indicates that the bulk of the oxygen-containing functional groups was removed from graphene oxide.

Graphene can be functionalized through noncovalent modification by wrapping with surfactants or through $\pi-\pi$ interaction with aromatic molecules such as 1pyrenebutanoic acid succinimidyl ester (PYBS) and the potassium salt of coronene tetracarboxylic acid (CS) [60]. In this procedure, the electronic structure of graphene remains unaffected. Water soluble graphene can be obtained by the non-covalent interaction of graphene with surfactants such as sodium dodecylsulphate (SDS) and cetyltrimethylammonium bromide (CTAB). Water soluble graphene can also be obtained when acidified graphene was treated with excess of polyethylene glycol (PEG) and concentrated $\mathrm{HCl}$ under solvothermal conditions [61].

Graphite was treated electrochemically to produce a colloidal suspension of chemically modified graphene. Liu et al. employed one step ionic liquid assisted electrochemical synthetic protocol to produce ionic liquid functionalized graphene sheets directly from graphene [33]. In this procedure, a commercial graphite electrode was used as the cathode, which was immersed in a phase-separated mixture of water and imidazolium-based ionic liquids. A constant potential of 10-20 V was applied across the electrodes. After $30 \mathrm{~min}$ electrochemical reaction, ionic liquid functionalized graphene sheets originating from the graphite anode. Ultrasonication of dried functionalized sheets in DMF resulted in a homogeneous dispersion. The average length and width of the ionic liquid functionalized graphene sheets were 700 and $500 \mathrm{~nm}$, respectively, as evidenced from the TEM images.
AFM image analysis revealed the thickness of the sheets to be $\sim 1.1 \mathrm{~nm}$.

4.2. Assembly at the Liquid-Liquid Interface. Molecular selfassembly of carbon nanostructures of various dimensions is of great interests due to their potential use in designing optically transparent conducting electrodes, solar cells, and other devices. Self-assembly of $\mathrm{C}_{60}$ nanosheets consists of hexagonal, rhombohedral, and mixed polygonal aggregates, which were synthesized by solvent engineering. Several groups created highly ordered monolayer films of graphene nanosheets at the liquid-liquid interface via molecular selfassembly. Chen et al. fabricated the self-assembled free standing membrane of $\mathrm{GO}$ at the liquid-air interface by evaporating the hydrosol of GO [62]. Layer-by-layer assembly has been employed by Kim and Min for the formation of nanofilms of reduced GO with multi-walled carbon nanotubes [63].

Ramesha and Sampath carried out an in situ Raman spectroelectrochemical study on GO films, which were assembled on conducting substrate such as gold by a layer-by-layer technique and subsequently reduced electrochemically [64].

Self-assembly has been identified for a long time as one of the most efficient strategies for "bottom-up" nanotechnology. Self-assembly of two-dimensional graphene sheets is an important strategy for producing macroscopic 3D graphene architectures for practical applications, such as thin films and layered paperlike materials. Recently, $\mathrm{Xu}$ et al. [65] synthesized self-assembled graphene hydrogel via a facile one-step hydrothermal method.

4.3. Surface Property. Graphene has extremely high specific surface area and high porosity, making them ideal for adsorption of different gases such as hydrogen $\left(\mathrm{H}_{2}\right)$, methane $\left(\mathrm{CH}_{4}\right)$, and carbon dioxide $\left(\mathrm{CO}_{2}\right)$. Theoretical calculations predict a large surface area of single-layer graphene close to $2600 \mathrm{~m}^{2} \mathrm{~g}^{-1}$ [66]. Experimentally, surface areas of fewlayer graphene sheets prepared by various methods have been measured using Brunauer-Emmett-Teller (BET) method and 
the surface areas are in the range of $270-1550 \mathrm{~m}^{2} \mathrm{~g}^{-1}$. Adsorption of $\mathrm{CO}_{2}$ by the graphene samples was measured at $195 \mathrm{~K}$ and $1 \mathrm{~atm}$. First-principle calculations show that $\mathrm{CO}_{2}$ molecules sit alternatively parallel configuration on the sixmembered rings [66]. Several groups studied the adsorption of $\mathrm{H}_{2}$ by graphene and other carbon nanomaterials [66-68]. It was found that the adsorption to be completely reversible in graphene and comparable to that of carbon nanotubes. First-principle calculations show that the $\mathrm{H}_{2}$ molecule sits in parallel and perpendicular orientations on the six-membered rings of graphene layer and that the single-layer graphene can uptake upto $7.7 \mathrm{wt} \%$ of hydrogen.

4.4. Fluorescence Quenching. Graphene has the ability of fluorescence quenching. This property of graphene can be utilized for the selective detection of biomolecules. The remarkable property of graphene of quenching fluorescence of aromatic molecules is associated with photoinduced electron transfer. Quenching of the fluorescence of porphyrin by graphene and photophysical properties of porphyrin-graphene complexes have been reported [69]. Ramakrishna and his group had studied the fluorescence quenching property of graphene [70]. Theoretical studies show that long-range energy transfer is associated in the fluorescence quenching of a dye molecule in the presence of graphene.

4.5. Raman Enhancement. The name surface enhanced Raman spectroscopy implies that it provides the same information that normal Raman spectroscopy does, simply with a greatly enhanced signal. The weak Raman signal can be greatly enhanced by the introduction of surfaceenhanced Raman scattering (SERS) spectroscopy in 1974 by Fleischmann et al. of the University of Southampton [71]. The introduction of surface-enhanced Raman spectroscopy (SERS) in 1974 has drawn a lot of attention to researchers due to the large enhancement of weak Raman signal and thereby facilitate convenient identification in chemical and biological systems. The exact mechanism of the enhancement effect of SERS is still a matter of controversy in the literature. There are two main mechanisms responsible for the large enhancement effect of weak Raman signal. One is the electromagnetic enhancement mechanism (EM) and the other is chemical enhancement mechanism (CM). The electromagnetic enhancement mechanism is based on the excitation of localized surface plasmons. On the other hand, the chemical enhancement mechanism relies on the charge transfer complex formation of the adsorbed molecule. However, it is very difficult to separate these two effects experimentally. SERS active substrate fabrication is a very important field in SERS research. The two most common SERS active substrates are metal colloids of coinage metals of $\mathrm{Au}, \mathrm{Ag}$, and $\mathrm{Cu}$ obtained from chemical reduction and the metal electrode surfaces roughened by one or more electrochemical oxidationreduction cycles. The recent advancement of nanotechnology has been utilized to fabricate various nanostructures from nanoparticles to nanowires, which can be used as SERS active substrates.
When SERS was invented, it turned out that only a few "free-electron-like" metals, mainly $\mathrm{Ag}, \mathrm{Au}$, and $\mathrm{Cu}$, can provide a large SERS effect for roughened metal surfaces and colloidal metal particles with dimension in the order of nanometers. However, lack of substrate generality limited the practical applications of SERS, while lack of surface generality restricted SERS studies to surfaces with ill-defined morphology that is unacceptable in surface science. Many groups had tried to overcome these two major limitation of SERS by obtaining SERS activity from other metallic surfaces other than $\mathrm{Ag}, \mathrm{Au}$, and $\mathrm{Cu}$ and from atomically flat (single crystal) surfaces. However, most of the metals used as SERS substrates have poor biological compatibility. Therefore, it is necessary to develop new novel substrates for Raman enhancement. To be an ideal SERS substrates, the material should be cheap, easy to obtain, and chemically inert as well as biocompatible. Recently, Professor Ling et al. and his co-workers at the Centre for Nanoscale Science and Technology, Peking University, Beijing, China had carried out the first systematic study to show that graphene can be used as a new efficient SERS active substrate with excellent biocompatibility and chemical inertness [72]. They used phthalocyanine $(\mathrm{Pc})$, rhodamine $6 \mathrm{G}(\mathrm{R} 6 \mathrm{G})$, protoporphyrin IX (PPP), and crystal violate (CV) as the common Raman probe molecules, which were deposited on graphene. They found that graphene has the potential as a substrate for Raman enhancement [72]. Furthermore, the Raman enhancement effect was investigated on different layers of graphene. The enhancement was found to be best on singlelayer graphene. The intensities of the Raman signals of probe molecules decreased roughly with the increase of the number of the graphene layers. Since the precise origin is unknown, further investigations are needed to be carry out. A Raman enhancement factor of 2-17 was obtained and the magnitude was found to be dependent on the vibrational symmetry of the modes of the molecules. Ling et al. [72] attributed this enhancement to the charge transfer between graphene and the probe molecules, which result in a chemical enhancement. It is important to mention here the EM mechanism requires rough surface, metal particles with large curvatures, and a surface which can absorb the incident light to generate surface plasmons. In case of CM mechanism, the distance between the probe molecule and the substrate must be below $0.2 \mathrm{~nm}$ and the Fermi level of the metal substrate matches symmetrically with the highest occupied molecular orbital (HOMO) and lowest unoccupied molecular orbital (LUMO) of the probe molecule to facilitate the charge transfer from metal to the molecule or vice versa. The surface of graphene is relatively smooth and highly optically transparent. The optical transmission through the graphene surface in the visible range is more than $95 \%$. In addition to this, the surface plasmon excitation on graphene is in the terahertz rather than in the visible range of electromagnetic spectrum. Due to these peculiar optical properties, graphene does not support electromagnetic enhancement mechanism. On the other hand, chemical enhancement mechanism is the only possibility for graphene in the enhancement of Raman signal. This consideration is supported by the fact that previous research work have already shown the 
possibility of charge transfer between graphene and some molecules [73]. If graphene can be used as an SERSactive substrate, it will provide an easy method to separate chemical enhancement mechanism from the electromagnetic enhancement mechanism

This is the first report about the Raman enhancement on the surface of graphene. In comparison to the common metals used as SERS substrates, graphene has many advantages such as easy preparation, lower cost, and biocompatibility. It is expected that this discovery will expand the application of graphene to microanalysis. In addition to the use of graphene as a potential bio/chemical sensors, this study is good for understanding the basic properties of graphene and SERS, such as the charge transfer of graphene and chemical enhancement mechanism (CM).

\section{Applications of Graphene}

The amazing properties of graphene has been explored for versatile applications ranging from electronic devices to electrode materials. It exhibits outstanding electronic properties, permitting electricity to flow rapidly through the materials. In fact, it has been shown that electrons in graphene behave as massless particles similar to photons, zipping across a graphene layer without scattering. This outstanding electronic property is crucial for many device applications and it is expected that graphene could eventually replace silicon $(\mathrm{Si})$ as the substance for computer chips, offering the prospect of ultrafast computers/quantum computers operating at terahertz speeds. Unfortunately, a thorough understanding of the electronic property of graphene has remained elusive.

Electron transport in graphene has been studied using the field-effect transistor (FET) configuration. In this configuration, a graphene sample is placed on an oxidized $\mathrm{Si}$ substrate and connected to source and drain electrode. The capacitance in these devices is dominated by the capacitance of the oxide layer, which makes it difficult to measure the quantum capacitance.

Capacitance is a fundamental property of a material which describes the material's ability to store energy in the form of charge. In classical physics, capacitance is limited by the repulsive electrical charges of electrons. However, another kind of capacitance exists and dominates the overall capacitance in a two dimensional material like graphene. The quantum capacitance is the result of Pauli exclusion principle, which states that two fermions, a class of common particles including electrons, protons, and neutrons cannot occupy the same energy level at the same time. Once a quantum state is filled, the subsequent fermions are forced to occupy successively higher energy states. The quantum capacitance measurements are an essential part to understand the extraordinary electronic property of graphene.

$\mathrm{Xia}$ and his co-workers at the Centre for Bioelectronics and Biosensors, Biodesign Institute of Arizona State University, USA described the first direct measurement of quantum capacitance of graphene in ionic liquid electrolytic media using a three electrode electrochemical gate configuration. Their findings were published in the August, 2010 issue of the journal Nature Nanotechnology [74]. In their study, two electrodes were attached to graphene, and a voltage applied across the material's two-dimensional surfaces by means of a third gate electrode. The use of an electrochemical gate and ionic liquid electrolyte 1-butyl-3-methyl imidazolium hexafluorophosphate $\left(\mathrm{BMIM}-\mathrm{PF}_{6}\right)$ in Tao's experiment reduced the Debye screening length to virtually zero, which makes the quantum capacitance a dominant factor of the measured capacitance. The quantum capacitance has a nonzero minimum at the Dirac point and a linear increase on both sides of the minimum with relatively small slopes. Tao's experimental results show that graphene's capacitance is very small. Due to high ionic concentration of the ionic liquid, the measured capacitance consists of interfacial capacitance due to the double layer formed at the graphene/ionic liquid interface and the quantum capacitance in series. The plot of quantum capacitance versus gate potential has a symmetric V-shape with a minimum at the Dirac point. It agrees with the theoretical model developed for ideal graphene and the measured absolute value of quantum capacitance are also close to the theoretical predictions. Their results strongly indicate that the interfacial capacitance in carbon-based electrodes has a quantum origin.

Tao's results have revealed the effect of charged impurities on the lowering of capacitance. Such low capacitance is desirable for applications in chemical and biosensors as it produces a lower signal-to-noise ratio, providing for extremely fine tuned resolution of chemical or biological agents. The sensitivity of graphene's single atomic layer geometry and low capacitance exhibit great potential for biosensor applications. Any biological substance that can interact with graphene's single atom surface layer can be detected, causing a large change in the electronic properties. One possible biosensor application under consideration is to study the antibody-antigen interaction by functionalizing the graphene's surface with antibodies.

5.1. Hydrogen Storage Material. Hydrogen has high energy density by mass. Therefore, it is considered as a key energy carriers. For practical applications, storage of hydrogen requires high gravimetric and volumetric densities, fast reaction kinetics, a low adsorption temperature, good reversibility, and low cost. However, safe storage of hydrogen is the biggest challenge due to the explosive nature of hydrogen. The most common methods of storing hydrogen are in the form of gas in pressurized cylinders, cryogenic liquid, or as adsorbed gas in solid materials. Currently, the high temperature dissolution of hydrogen in metal hydrides poses a problem. Therefore, storage of hydrogen in materials by chemical process is indeed a safer method. Recent advancement in nanomaterials can play an important role for storage applications due to the large surface-to-volume ratio and decrease of hydrogen diffusion path lengths in nanomaterials. In this regard, the use of various transition metal nanoparticles such as palladium $(\mathrm{Pd})$, platinum $(\mathrm{Pt})$, and titanium $(\mathrm{Ti})$ deposited on carbon supports have been investigated for their hydrogen uptake capability. Graphene can be an ideal material for hydrogen storage owing to the light weight, high surface area, 
TABLE 3: A comparison of various carbon electrode materials for supercapacitors.

\begin{tabular}{|c|c|c|c|c|c|c|}
\hline \multirow{2}{*}{ Carbon-based material } & \multirow{2}{*}{$\begin{array}{l}\text { Specific surface } \\
\text { area }\left(\mathrm{m}^{2} \mathrm{~g}^{-1}\right)\end{array}$} & \multirow{2}{*}{ Density $\left(\mathrm{g} \mathrm{cm}^{-3}\right)$} & \multirow{2}{*}{$\begin{array}{l}\text { Electrical conductivity } \\
\qquad\left(\mathrm{S} \mathrm{cm}^{-1}\right)\end{array}$} & \multirow[b]{2}{*}{ Cost } & \multicolumn{2}{|c|}{ Specific capacitance $\left(\mathrm{Fg}^{-1}\right)$} \\
\hline & & & & & $\begin{array}{l}\text { Aqueous } \\
\text { electrolyte }\end{array}$ & $\begin{array}{l}\text { Organic } \\
\text { electrolyte }\end{array}$ \\
\hline Fullerene & $1100-1400$ & 1.72 & $10^{-8}-10^{-14}$ & Medium & - & - \\
\hline CNTs & $120-500$ & 0.6 & $10^{4}-10^{5}$ & High & $50-100$ & $<60$ \\
\hline Graphene & 2630 & $>1$ & $10^{6}$ & High & $100-200$ & $80-110$ \\
\hline Graphite & 10 & 2.26 & $10^{4}$ & Low & & \\
\hline $\begin{array}{l}\text { Activated } \\
\text { carbon }\end{array}$ & $1000-3500$ & $0.4-0.7$ & $0.1-1$ & Low & $<200$ & $<100$ \\
\hline Templated porous carbon & $500-3000$ & $0.5-1$ & $0.3-10$ & High & $120-350$ & $60-140$ \\
\hline Activated carbon fibers & $1000-3000$ & $0.3-0.8$ & $5-10$ & Medium & $120-370$ & $80-200$ \\
\hline Carbon aerogels & $400-1000$ & $0.5-0.7$ & $1-10$ & Low & $100-125$ & $<80$ \\
\hline
\end{tabular}

and chemical stability. Hydrogen can be chemically stored in graphene by physisorption or chemisorption. However, practically it exhibits a low hydrogen storage capacity at ambient temperatures and moderate pressures. For example, a single-layer graphene was shown to store $0.4 \mathrm{wt} \%$ hydrogen at $100 \mathrm{kPa}$ pressure and cryogenic temperature. Therefore, chemical modification of carbon supports by metal or metal oxide nanoparticles is essential to enhance the hydrogen uptake efficiency. Pd has strong affinity towards hydrogen adsorption. The role of Pd nanoparticles on graphene can be enhanced by three times under moderate temperature and pressure. Therefore, graphene and its nanocomposites have a great potential in hydrogen storage, which can be employed in fuel cell technology with the fuel cell serving as the fuel source. Recently, Maidhily et al. [75] have utilized the functionalized graphene sheets as catalyst support for the proton exchange membrane fuel cells. Subrahmanyam and his group studied the chemical storage of hydrogen in few layer graphene [76].

5.2. Actuators. The extraordinary mechanical, optical, and electrical properties of graphene have been exploited by many scientists to develop actuators. Park et al. designed a bilayer paper composed of adjacent graphene oxide and multi-walled carbon nanotube layers and demonstrated a macroscopic graphene-based actuators [77]. The papers curl depending on the humidity and/or temperature. Xie et al. [78] developed a graphene film actuator by asymmetric surface modification of two opposite sides of monolithic graphene film with hexane and oxygen plasma, respectively. The actuation motion was induced through asymmetric charging and discharging. A bimorph microactuator has been developed based on graphene-on-organic hybrid film. The graphene-on-organic film actuator generates a flapping and bending motion that can be controlled by varying the frequency and duration of the applied potential. Recently, $\mathrm{Wu}$ et al. developed a remote-controlled transparent, large area grapheme-based robot [79]. This grapheme-based actuator can curl and uncurl in the absence and presence of infrared (IR) radiation [79]. This remote-controlled based robot picks up any object, moves it to a desired location, and drops it by the remote control of IR radiation. This is undoubtedly an elegant demonstration of photothermal energy transformation by grapheme, based actuators.

5.3. Supercapacitors or Ultracapacitors. Electrochemical capacitors, also called supercapacitors or ultracapacitors, store energy using either ion adsorption (electrochemical doublelayer capacitors) or fast surface redox reactions or faradaic reactions (pseudocapacitors/redox capacitors). They can complement or replace batteries in electrical energy storage and harvesting applications, when high power delivery or uptake is needed. A notable improvement in performance has been achieved through recent advances in understanding charge storage mechanisms and the development of advanced nanostructured materials. While a battery is a high energy and low power device, extensively used in conventional applications, the supercapacitor acts as a low energy and high power device and is ideal for use in high power pulse requirements. Unlike a battery, supercapacitors possess a high power density and longer cycle life. They fill the gap between batteries and conventional dielectric capacitors. In the literature, three different kinds of supercapacitors based on carbon-carbon, transition metal oxides and conducting polymers have been reported.

The main factor to obtain high capacitance value by charging the double layer is in using high specific surface area, blocking and electronically conducting electrodes. Graphitic carbon satisfies all the requirements for supercapacitor applications such as high conductivity, electrochemical stability, and open porosity. Activated, template, and carbide derived carbons, carbon fabrics, fibres, nanotubes, and nanohorns have been tested for electrochemical doublelayer capacitor applications. Activated carbons are the most widely used electrode materials due to their high specific surface area, low cost, and easy processability.

Table 3 summarizes some properties and characteristics of various carbon electrode materials for supercapacitors. Graphene-based materials, including zero-dimensional fullerenes, one-dimensional carbon nanotubes (CNTs), twodimensional grapheme, and three-dimensional graphite are of particular interest due to their exceptional electrical and mechanical properties and unique structures. Recent researches on electrode materials have been mainly focused 
on the carbon nanostructures like carbon nanotubes (CNTs). CNTs with excellent electrical conductivity and high surface areas have been fabricated for supercapacitors since late 90s. However, CNT-based supercapacitors have not met the expected performance due to the observed contact resistance between the electrode and current collector and also due to the high cost. Hence, many studies have been carried out to improve the performance of CNT-based supercapacitors by growing CNTs directly on bulk metals to remove the contact resistance [80]. Recently, grapheme-based electrode material has been used for supercapacitor applications [81] and two-graphene supercapacitor application studies had been reported with the specific capacitance of $117 \mathrm{~F} \mathrm{~g}^{-1}$ in aqueous $\mathrm{H}_{2} \mathrm{SO}_{4}$ [81] and $135 \mathrm{~F} \mathrm{~g}^{-1}$ in aqueous electrolyte [82] based on a multilayered graphene material. In contrast to the conventional high surface materials, the effective surface area of grapheme-based materials as capacitor electrode materials does not depend on the distribution of pores at the solid state [82], which is different from the current supercapacitors fabricated with activated carbons and carbon nanotubes. Obviously, the effective surface area of graphene materials should depend highly on the layers. Therefore, the single- or few-layered graphene should be expected to exhibit higher effective surface area and thus better supercapacitor performance. Wang et al. [8] fabricated supercapacitor devices using graphene as electrode materials and investigated their performance. The single-layered graphene oxide sheets were reduced using gas-based hydrazine reduction at room temperature. The reduced graphene materials produced by this method have a lower degree of agglomeration than the chemically modified graphene prepared in aqueous solution at the high temperature. A maximum specific capacitance of $205 \mathrm{~F} \mathrm{~g}^{-1}$ at $1.0 \mathrm{~V}$ in aqueous electrolyte with energy density of $28.5 \mathrm{~W} \mathrm{~h} \mathrm{~kg}^{-1}$ has been obtained, which are the best results for graphene materials so far and also significantly higher than those of CNT-based supercapacitors. Furthermore, the power density of the capacitors reaches as high as $10 \mathrm{~kW} \mathrm{~kg}^{-1}$, higher than that for CNT-based supercapacitors. Figure 3 illustrates the optical image of an industry-grade coin-shaped graphene-based supercapacitor device.

Subsequent development of grapheme-based nanocomposites using conducting polymers is an important step of improvisation in the area of nanoscience and nanotechnology. This kind of graphene-based polymer nanocomposites can be used as electrode material in supercapacitors. The main conductive polymer materials that have been investigated for the supercapacitor electrode are polyaniline (PANI), polypyrrole (PPY), polythiophene (PTH), and their derivatives.

Freestanding and flexible graphene/polyaniline composite paper was prepared by Wang et al. [83] employing an in situ anodic electropolymerization of polyaniline film on graphene paper. This graphene-based composite paper electrode, consisting of graphene/polyaniline composite sheets as building blocks, shows a favorable tensile strength of $12.6 \mathrm{MPa}$ and exhibits a stable large electrochemical capacitance $\left(233 \mathrm{~F} \mathrm{~g}^{-1}\right.$ and $135 \mathrm{~F} \mathrm{~cm}^{-3}$ for gravimetric and volumetric capacitances). These values are much larger compared to many other currently available carbon-based

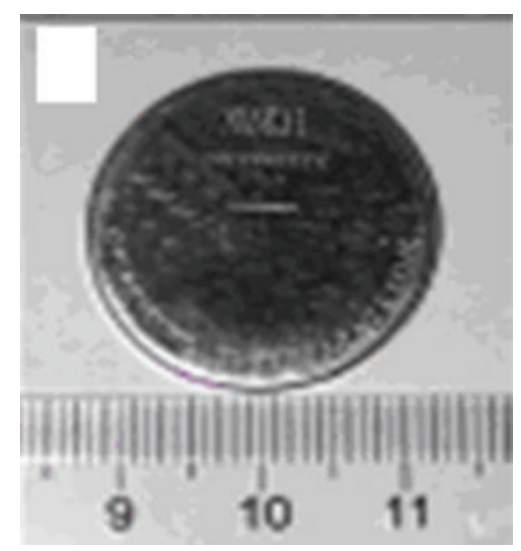

FIGURE 3: The optical image of an industry-grade coin-shaped graphene-based supercapacitor device. Reprinted with permission from Wang et al. [8] Copyright (2009) by American Chemical Society.

flexible electrodes and is therefore particularly promising for flexible supercapacitors.

\section{Future Prospects of Graphene}

It is expected that the remarkable electronic property of graphene will bring a new era in nanoelectronics. Despite the high expectation about graphene-based electronics, "graphenium" microprocessors are unlikely to appear for the next few years. However, many other graphene-based applications are likely to be utilized.

The most direct application for graphene is probably its use in composite materials. Indeed, it has been demonstrated that by spreading a small amount of graphene throughout polymers, tough lightweight materials can be designed. The composites conduct electricity and can withstand much higher temperatures than the polymers. Recently, a mechanical engineering professor at Northwestern University, USA synthesized graphene-based polymer composite materials. These grapheme-based polymer composites could be ideal to make lightweight gasoline tanks and plastic containers. These composites can be potentially used to make lighter, more fuel-efficient aircraft and car parts, stronger wind turbines, medical implants, and sports equipments.

Another option is the use of graphene powder in electric batteries that are already one of the main markets for graphite. A high surface-to-volume ratio and high conductivity provided by graphene powder can lead to improvements in the overall efficiency of batteries, taking over from the carbon nanofibers used in modern batteries.

High conductivity and high optical transparency make graphene suitable for fabricating transparent conducting coating in LCDs and solar cells. Recently, researchers from Korea and USA developed ultraviolet (UV) nitride lightemitting diode which uses a few layer of graphene as a transparent conducting layer.

The outstanding electronic property of graphene is crucial for many device applications and it is expected 
that graphene could eventually replace silicon $(\mathrm{Si})$ as the substance for computer chips, offering the prospect of ultrafast computers/quantum computers operating at terahertz speeds.

\section{Conclusions}

The discovery of graphene in 2004 triggered research activities at various experimental and theoretical aspects worldwide due to its extremely large surface area and unique material properties [84]. Within few years of discovery, grapheme-based materials and their composites enjoy the status of being the new supermaterial on the horizon of material science and condensed matter physics owing to their versatile applications in various fields. Different protocols have been employed for the synthesis of graphene. Stable dispersions can be formed by functionalization of graphene. This paper summarizes the background on the discovery of graphene followed by the development in synthesis, functionalization, and applications in various fields from sensors to energy-related applications. It is expected that by 2015, the global market of graphene-based products would reach $\$ 67$ Million.

\section{Acknowledgments}

The author would like to acknowledge financial support from the project funded by the UGC, New Delhi (Grant no. PSW-038/10-11-ERO). The author would like to thank Dr. Balaprasad Ankamwar and Professor Tapan Ganguly for discussion.

\section{References}

[1] H. W. Kroto, J. R. Heath, S. C. O'Brien, R. F. Curl, and R. E. Smalley, “C60: Buckminsterfullerene," Nature, vol. 318, no. 6042, pp. 162-163, 1985.

[2] S. Iijima, "Helical microtubules of graphitic carbon," Nature, vol. 354, no. 6348, pp. 56-58, 1991.

[3] K. S. Novoselov, A. K. Geim, S. V. Morozov et al., "Electric field in atomically thin carbon films," Science, vol. 306, no. 5696, pp. 666-669, 2004.

[4] A. K. Geim and K. S. Novoselov, "The rise of graphene," Nature Materials, vol. 6, no. 3, pp. 183-191, 2007.

[5] C. N. R. Rao, A. K. Sood, K. S. Subrahmanyam, and A. Govindaraj, "Graphene: the new two-dimensional nanomaterial," Angewandte Chemie, vol. 48, no. 42, pp. 7752-7777, 2009.

[6] K. S. Novoselov, Z. Jiang, Y. Zhang et al., "Room-temperature quantum hall effect in graphene," Science, vol. 315, no. 5817, p. 1379, 2007.

[7] A. K. Geim, "Graphene: status and prospects," Science, vol. 324, no. 5934, pp. 1530-1534, 2009.

[8] Y. Wang, Z. Shi, Y. Huang et al., "Supercapacitor devices based on graphene materials," Journal of Physical Chemistry C, vol. 113, no. 30, pp. 13103-13107, 2009.

[9] D. V. Kosynkin, A. L. Higginbotham, A. Sinitskii et al., "Longitudinal unzipping of carbon nanotubes to form graphene nanoribbons," Nature, vol. 458, no. 7240, pp. 872-876, 2009.

[10] D. B. Shinde, J. Debgupta, A. Kushwaha, M. Aslam, and V. K. Pillai, "Electrochemical unzipping of multi-walled carbon nanotubes for facile synthesis of high-quality graphene nanoribbons," Journal of the American Chemical Society, vol. 133, no. 12, pp. 4168-4171, 2011.

[11] X. Li, G. Zhang, X. Bai et al., "Highly conducting graphene sheets and Langmuir-Blodgett films," Nature Nanotechnology, vol. 3, no. 9, pp. 538-542, 2008.

[12] V. C. Tung, M. J. Allen, Y. Yang, and R. B. Kaner, "Highthroughput solution processing of large-scale graphene," Nature Nanotechnology, vol. 4, no. 1, pp. 25-29, 2009.

[13] M. Choucair, P. Thordarson, and J. A. Stride, "Gram-scale production of graphene based on solvothermal synthesis and sonication," Nature Nanotechnology, vol. 4, no. 1, pp. 30-33, 2009.

[14] X. Yang, X. Dou, A. Rouhanipour, L. Zhi, H. J. Räder, and K. Müllen, "Two-dimensional graphene nanoribbons," Journal of the American Chemical Society, vol. 130, no. 13, pp. 4216-4217, 2008.

[15] Y. Hernandez, V. Nicolosi, M. Lotya et al., "High-yield production of graphene by liquid-phase exfoliation of graphite," Nature Nanotechnology, vol. 3, no. 9, pp. 563-568, 2008.

[16] A. Reina, X. Jia, J. Ho et al., "Large area, few-layer graphene films on arbitrary substrates by chemical vapor deposition," Nano Letters, vol. 9, no. 1, pp. 30-35, 2009.

[17] K. V. Emtsev, A. Bostwick, K. Horn et al., "Towards wafersize graphene layers by atmospheric pressure graphitization of silicon carbide," Nature Materials, vol. 8, no. 3, pp. 203-207, 2009.

[18] S. Park and R. S. Ruoff, "Chemical methods for the production of graphenes," Nature Nanotechnology, vol. 4, no. 4, pp. 217224, 2009.

[19] D. Li, M. B. Müller, S. Gilje, R. B. Kaner, and G. G. Wallace, "Processable aqueous dispersions of graphene nanosheets," Nature Nanotechnology, vol. 3, no. 2, pp. 101-105, 2008.

[20] S. Stankovich, D. A. Dikin, G. H. B. Dommett et al., "Graphene-based composite materials," Nature, vol. 442, no. 7100, pp. 282-286, 2006.

[21] S. Gilje, S. Han, M. Wang, K. L. Wang, and R. B. Kaner, "A chemical route to graphene for device applications," Nano Letters, vol. 7, no. 11, pp. 3394-3398, 2007.

[22] S. Stankovich, D. A. Dikin, R. D. Piner et al., "Synthesis of graphene-based nanosheets via chemical reduction of exfoliated graphite oxide," Carbon, vol. 45, no. 7, pp. 15581565, 2007.

[23] Y. Si and E. T. Samulski, "Synthesis of water soluble graphene," Nano Letters, vol. 8, no. 6, pp. 1679-1682, 2008.

[24] W. Chen, L. Yan, and P. R. Bangal, "Preparation of graphene by the rapid and mild thermal reduction of graphene oxide induced by microwaves," Carbon, vol. 48, no. 4, pp. 1146$1152,2010$.

[25] D. A. Sokolov, K. R. Shepperd, and T. M. Orlando, "Formation of graphene features from direct laser-induced reduction of graphite oxide," Journal of Physical Chemistry Letters, vol. 1, no. 18, pp. 2633-2636, 2010.

[26] M. Baraket, S. G. Walton, Z. Wei, E. H. Lock, J. T. Robinson, and P. Sheehan, "Reduction of graphene oxide by electron beam generated plasmas produced in methane/argon mixtures," Carbon, vol. 48, no. 12, pp. 3382-3390, 2010.

[27] K. Vinodgopal, B. Neppolian, I. V. Lightcap, F. Grieser, M. Ashokkumar, and P. V. Kamat, "Sonolytic design of graphene$\mathrm{Au}$ nanocomposites. simultaneous and sequential reduction of graphene oxide and Au(III)," Journal of Physical Chemistry Letters, vol. 1, no. 13, pp. 1987-1993, 2010. 
[28] Y. Zhou, Q. Bao, L. A. L. Tang, Y. Zhong, and K. P. Loh, "Hydrothermal dehydration for the "green" reduction of exfoliated graphene oxide to graphene and demonstration of tunable optical limiting properties," Chemistry of Materials, vol. 21, no. 13, pp. 2950-2956, 2009.

[29] W. Li, M. Zhao, X. Zhao, Y. Xia, and Y. Mu, "Hydrogen saturation stabilizes vacancy-induced ferromagnetic ordering in graphene," Physical Chemistry Chemical Physics, vol. 12, no. 41, pp. 13699-13706, 2010.

[30] Z. Wang, X. Zhou, J. Zhang, F. Boey, and H. Zhang, "Direct electrochemical reduction of single-layer graphene oxide and subsequent functionalization with glucose oxidase," Journal of Physical Chemistry C, vol. 113, no. 32, pp. 14071-14075, 2009.

[31] G. Wang, B. Wang, J. Park, Y. Wang, B. Sun, and J. Yao, "Highly efficient and large-scale synthesis of graphene by electrolytic exfoliation," Carbon, vol. 47, no. 14, pp. 3242-3246, 2009.

[32] S. Liu, J. Ou, J. Wang, X. Liu, and S. Yang, "A simple two-step electrochemical synthesis of graphene sheets film on the ITO electrode as supercapacitors," Journal of Applied Electrochemistry, pp. 1-4, 2011.

[33] N. Liu, F. Luo, H. Wu, Y. Liu, C. Zhang, and J. Chen, "Onestep ionic-liquid-assisted electrochemical synthesis of ionicliquid-functionalized graphene sheets directly from graphite," Advanced Functional Materials, vol. 18, no. 10, pp. 1518-1525, 2008.

[34] G. Ruan, Z. Sun, Z. Peng, and J. M. Tour, "Growth of graphene from food, insects, and waste," ACS Nano, vol. 5, no. 9, pp. 7601-7607, 2011.

[35] P. R. Wallace, “The band theory of graphite," Physical Review, vol. 71, pp. 622-634, 1947.

[36] R. E. Peierls, "Quelques proprietes typiques des corpses solides," Annales de l'Institut Henri Poincaré, vol. 5, pp. 177222, 1935.

[37] L. D. Landau, "Zur Theorie der phasenumwandlungen II," Physikalische Zeitschrift der Sowjetunion, vol. 11, pp. 26-35, 1937.

[38] J. W. Evans, P. A. Thiel, and M. C. Bartelt, "Morphological evolution during epitaxial thin film growth: formation of 2D islands and 3D mounds," Surface Science Reports, vol. 61, no. 1-2, pp. 1-128, 2006.

[39] K. S. Novoselov, D. Jiang, F. Schedin et al., "Two-dimensional atomic crystals," Proceedings of the National Academy of Sciences of the United States of America, vol. 102, no. 30, pp. 10451-10453, 2005.

[40] K. A. Ritter and J. W. Lyding, "Characterization of nanometersized, mechanically exfoliated graphene on the H-passivated Si(100) surface using scanning tunneling microscopy," Nanotechnology, vol. 19, no. 1, Article ID 015704, 2008.

[41] P. W. Sutter, J. I. Flege, and E. A. Sutter, "Epitaxial graphene on ruthenium," Nature Materials, vol. 7, no. 5, pp. 406-411, 2008.

[42] K. S. Kim, Y. Zhao, H. Jang et al., "Large-scale pattern growth of graphene films for stretchable transparent electrodes," Nature, vol. 457, no. 7230, pp. 706-710, 2009.

[43] J. Wang, M. Zhu, R. A. Outlaw, X. Zhao, D. M. Manos, and B. C. Holloway, "Synthesis of carbon nanosheets by inductively coupled radio-frequency plasma enhanced chemical vapor deposition," Carbon, vol. 42, no. 14, pp. 2867-2872, 2004.

[44] W. S. Hummers and R. E. Offeman, "Preparation of graphitic oxide," Journal of the American Chemical Society, vol. 80, no. 6, p. $1339,1958$.

[45] N. I. Kovtyukhova, "Layer-by-layer assembly of ultrathin composite films from micron-sized graphite oxide sheets and polycations," Chemistry of Materials, vol. 11, no. 3, pp. 771$778,1999$.
[46] G. Wang, J. Yang, J. Park et al., "Facile synthesis and characterization of graphene nanosheets," Journal of Physical Chemistry C, vol. 112, no. 22, pp. 8192-8195, 2008.

[47] C. N. R. Rao, K. Biswas, K. S. Subrahmanyam, and A. Govindaraj, "Graphene, the new nanocarbon," Journal of Materials Chemistry, vol. 19, no. 17, pp. 2457-2469, 2009.

[48] J. Zhang, H. Yang, G. Shen, P. Cheng, J. Zhang, and S. Guo, "Reduction of graphene oxide vial-ascorbic acid," Chemical Communications, vol. 46, no. 7, pp. 1112-1114, 2010.

[49] C. Zhu, S. Guo, Y. Fang, and S. Dong, "Reducing sugar: new functional molecules for the green synthesis of graphene nanosheets," ACS Nano, vol. 4, no. 4, pp. 2429-2437, 2010.

[50] E. C. Salas, Z. Sun, A. Lüttge, and J. M. Tour, "Reduction of graphene oxide via bacterial respiration," ACS Nano, vol. 4, no. 8, pp. 4852-4856, 2010.

[51] O. E. Andersson, B. L. V. Prasad, H. Sato et al., "Structure and electronic properties of graphite nanoparticles," Physical Review B, vol. 58, no. 24, pp. 16387-16395, 1998.

[52] B. L. V. Prasad, H. Sato, T. Enoki et al., "Heat-treatment effect on the nanosized graphite $\pi$-electron system during diamond to graphite conversion," Physical Review B, vol. 62, no. 16, pp. 11209-11218, 2000.

[53] K. S. Subrahmanyam, L. S. Panchakarla, A. Govindaraj, and C. N. R. Rao, "Simple method of preparing graphene flakes by an arc-discharge method," Journal of Physical Chemistry C, vol. 113 , no. 11, pp. 4257-4259, 2009.

[54] W. Gao, L. B. Alemany, L. Ci, and P. M. Ajayan, "New insights into the structure and reduction of graphite oxide," Nature Chemistry, vol. 1, no. 5, pp. 403-408, 2009.

[55] P. K. Ang, S. Wang, Q. Bao, J. T. L. Thong, and K. P. Loh, "High-throughput synthesis of graphene by intercalationexfoliation of graphite oxide and study of ionic screening in graphene transistor," ACS Nano, vol. 3, no. 11, pp. 3587-3594, 2009.

[56] M. Lotya, Y. Hernandez, P. J. King et al., "Liquid phase production of graphene by exfoliation of graphite in surfactant/water solutions," Journal of the American Chemical Society, vol. 131, no. 10, pp. 3611-3620, 2009.

[57] C. N. R. Rao, K. S. Subrahmanyam, H. S. S. Ramakrishna Matte, and A. Govindaraj, "Graphene: synthesis, functionalization and properties," Modern Physics Letters B, vol. 25, no. 7, pp. 427-451, 2011.

[58] S. Niyogi, E. Bekyarova, M. E. Itkis, J. L. McWilliams, M. A. Hamon, and R. C. Haddon, "Solution properties of graphite and graphene," Journal of the American Chemical Society, vol. 128, no. 24, pp. 7720-7721, 2006.

[59] K. A. Worsley, P. Ramesh, S. K. Mandal, S. Niyogi, M. E. Itkis, and R. C. Haddon, "Soluble graphene derived from graphite fluoride," Chemical Physics Letters, vol. 445, no. 1-3, pp. 5156, 2007.

[60] A. Ghosh, K. V. Rao, S. J. George, and C. N. R. Rao, "Noncovalent functionalization, exfoliation, and solubilization of graphene in water by employing a fluorescent coronene carboxylate," Chemistry, vol. 16, no. 9, pp. 2700-2704, 2010.

[61] K. S. Subrahmanyam, S. R. C. Vivekchand, A. Govindaraj, and C. N. R. Rao, "A study of graphenes prepared by different methods: characterization, properties and solubilization," Journal of Materials Chemistry, vol. 18, no. 13, pp. 1517-1523, 2008.

[62] C. Chen, Q. H. Yang, Y. Yang et al., "Self-assembled freestanding graphite oxide membrane," Advanced Materials, vol. 21, no. 29, pp. 3007-3011, 2009. 
[63] Y. K. Kim and D. H. Min, "Durable large-area thin films of graphene/carbon nanotube double layers as a transparent electrode," Langmuir, vol. 25, no. 19, pp. 11302-11306, 2009.

[64] G. K. Ramesha and N. S. Sampath, "Electrochemical reduction of oriented Graphene oxide films: an in situ Raman spectroelectrochemical study," Journal of Physical Chemistry C, vol. 113, no. 19, pp. 7985-7989, 2009.

[65] Y. Xu, K. Sheng, C. Li, and G. Shi, "Self-assembled graphene hydrogel via a one-step hydrothermal process," ACS Nano, vol. 4, no. 7, pp. 4324-4330, 2010.

[66] A. Ghosh, K. S. Subrahmanyam, K. S. Krishna et al., "Uptake of $\mathrm{H}_{2}$ and $\mathrm{CO}_{2}$ by graphene," Journal of Physical Chemistry $C$, vol. 112, no. 40, pp. 15704-15707, 2008.

[67] G. Gundiah, A. Govindaraj, N. Rajalakshmi, K. S. Dhathathreyan, and C. N. R. Rao, "Hydrogen storage in carbon nanotubes and related materials," Journal of Materials Chemistry, vol. 13, no. 2, pp. 209-213, 2003.

[68] D. J. Collins and H. C. Zhou, "Hydrogen storage in metalorganic frameworks," Journal of Materials Chemistry, vol. 17, no. 30, pp. 3154-3160, 2007.

[69] Y. Xu, L. Zhao, H. Bai, W. Hong, C. Li, and G. Shi, "Chemically converted graphene induced molecular flattening of 5,10,15,20-tetrakis(1-methyl-4-pyridinio)porphyrin and its application for optical detection of cadmium(II) ions," Journal of the American Chemical Society, vol. 131, no. 37, pp. 1349013497, 2009.

[70] H. S. S. Ramakrishna Matte, K. S. Subrahmanyam, K. Venkata Rao, S. J. George, and C. N. R. Rao, "Quenching of fluorescence of aromatic molecules by graphene due to electron transfer," Chemical Physics Letters, vol. 506, no. 4-6, pp. 260-264, 2011.

[71] M. Fleischmann, P. J. Hendra, and A. J. McQuillan, "Raman spectra of pyridine adsorbed at a silver electrode," Chemical Physics Letters, vol. 26, no. 2, pp. 163-166, 1974.

[72] X. Ling, L. Xie, Y. Fang et al., "Can graphene be used as a substrate for Raman enhancement?" Nano Letters, vol. 10, no. 2, pp. 553-561, 2010.

[73] A. K. Manna and S. K. Pati, "Tuning the electronic structure of graphene by molecular charge transfer: a computational study," Chemistry, vol. 4, no. 6, pp. 855-860, 2009.

[74] J. Xia, F. Chen, J. Li, and N. Tao, "Measurement of the quantum capacitance of graphene," Nature Nanotechnology, vol. 4, no. 8, pp. 505-509, 2009.

[75] M. Maidhily, N. Rajalakshmi, and K. S. Dhathathreyan, "Electrochemical impedance diagnosis of micro porous layer in polymer electrolyte membrane fuel cell electrodes," International Journal of Hydrogen Energy, vol. 36, no. 19, pp. 12352 12360, 2011.

[76] K. S. Subrahmanyam, P. Kumar, U. Maitra et al., "Chemical storage of hydrogen in few-layer graphene," Proceedings of the National Academy of Sciences of the United States of America, vol. 108, no. 7, pp. 2674-2677, 2011.

[77] S. Park, J. An, J. W. Suk, and R. S. Ruoff, "Graphene-based actuators," Small, vol. 6, no. 2, pp. 210-212, 2010.

[78] X. Xie, L. Qu, C. Zhou et al., "An asymmetrically surfacemodified graphene film electrochemical actuator," ACS Nano, vol. 4, no. 10, pp. 6050-6054, 2010.

[79] C. Wu, J. Feng, L. Peng et al., "Large-area graphene realizing ultrasensitive photothermal actuator with high transparency: new prototype robotic motions under infrared-light stimuli," Journal of Materials Chemistry, vol. 21, no. 46, pp. 1858418591, 2011.
[80] S. Talapatra, S. Kar, S. K. Pal et al., "Direct growth of aligned carbon nanotubes on bulk metals," Nature Nanotechnology, vol. 1, no. 2, pp. 112-116, 2006.

[81] S. R. C. Vivekchand, C. S. Rout, K. S. Subrahmanyam, A. Govindaraj, and C. N. R. Rao, "Graphene-based electrochemical supercapacitors," Journal of Chemical Sciences, vol. 120, no. 1, pp. 9-13, 2008.

[82] M. D. Stoller, S. Park, Z. Yanwu, J. An, and R. S. Ruoff, "Graphene-Based ultracapacitors," Nano Letters, vol. 8, no. 10, pp. 3498-3502, 2008.

[83] D. W. Wang, F. Li, J. Zhao et al., "Fabrication of graphene/polyaniline composite paper via in situ anodic electropolymerization for high-performance flexible electrode," ACS Nano, vol. 3, no. 7, pp. 1745-1752, 2009.

[84] M. J. Allen, V. C. Tung, and R. B. Kaner, "Honeycomb carbon: a review of graphene," Chemical Reviews, vol. 110, no. 1, pp. 132-145, 2010. 


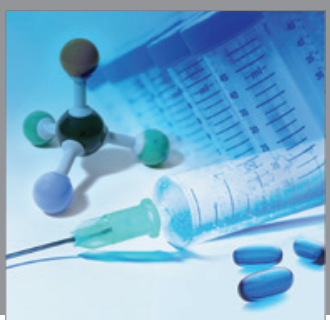

International Journal of

Medicinal Chemistry

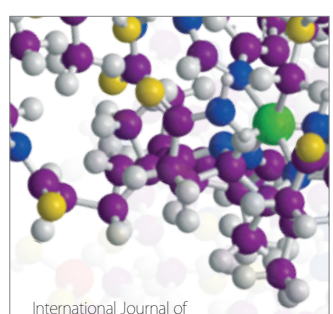

Carbohydrate Chemistry

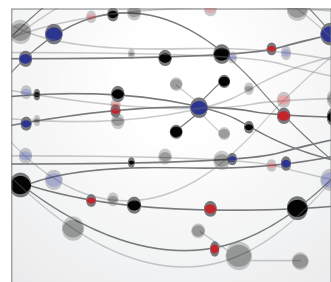

The Scientific World Journal
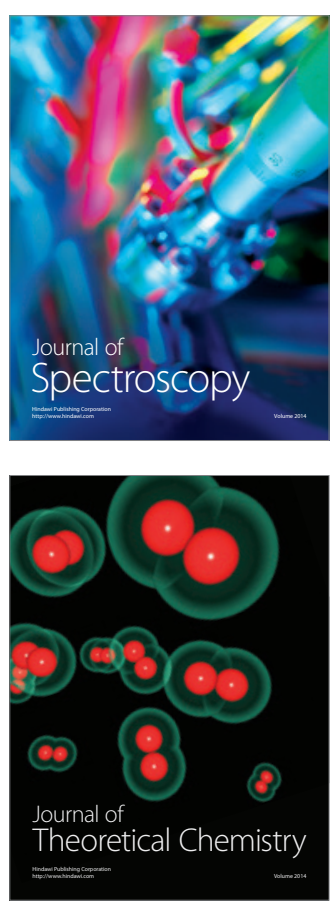
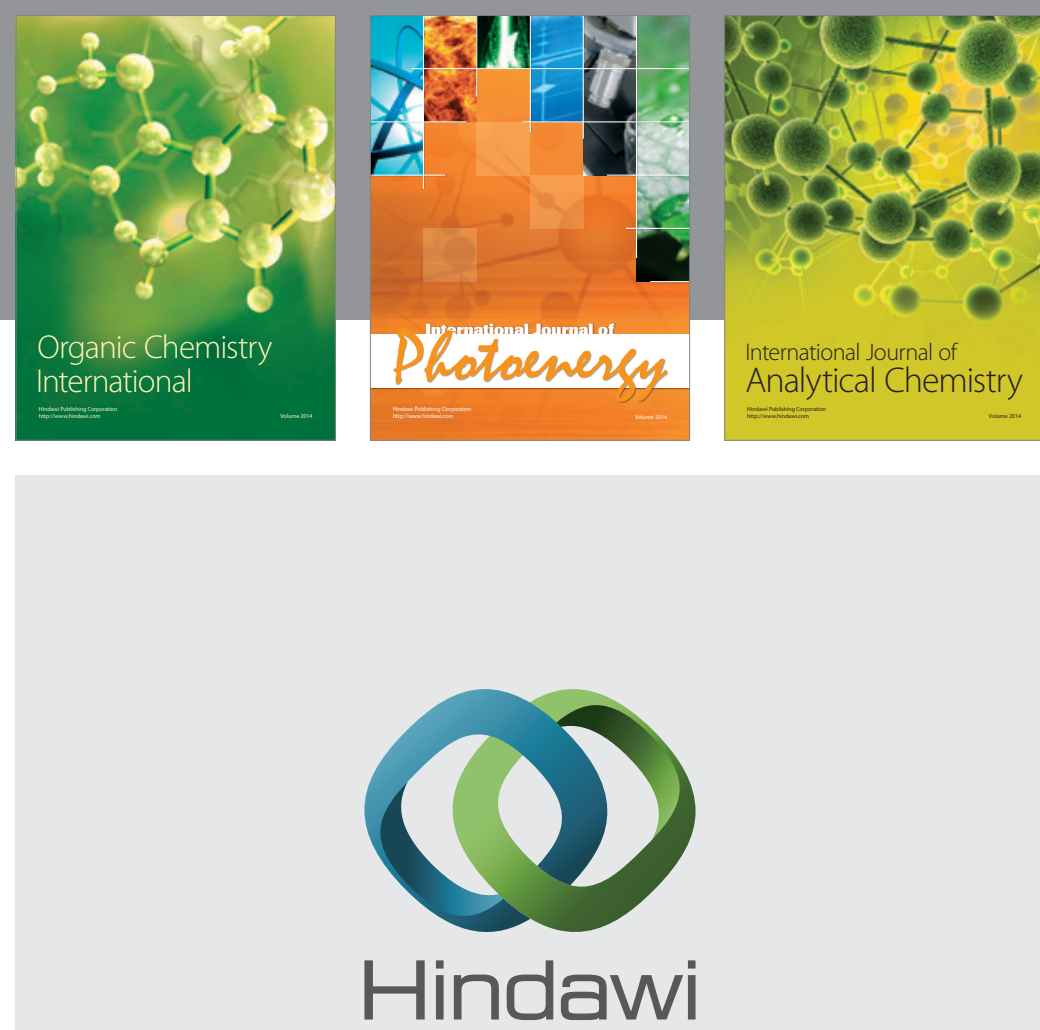

Submit your manuscripts at

http://www.hindawi.com
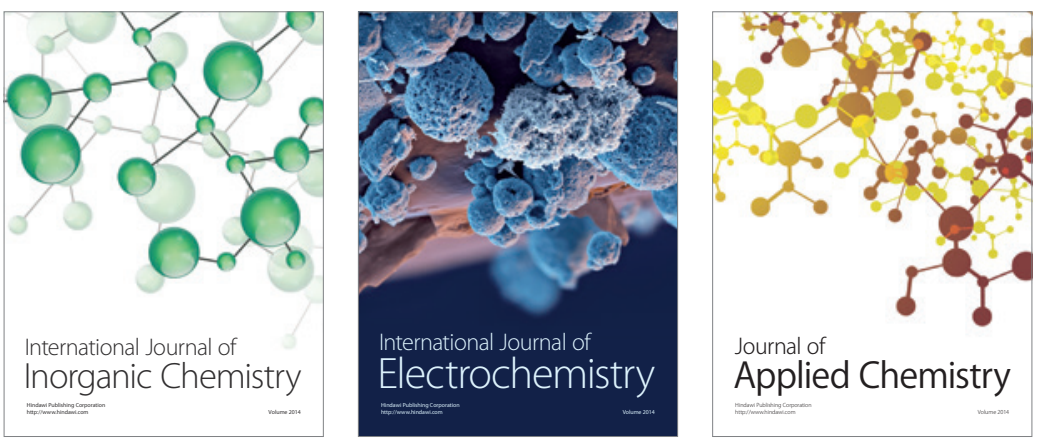

Journal of

Applied Chemistry
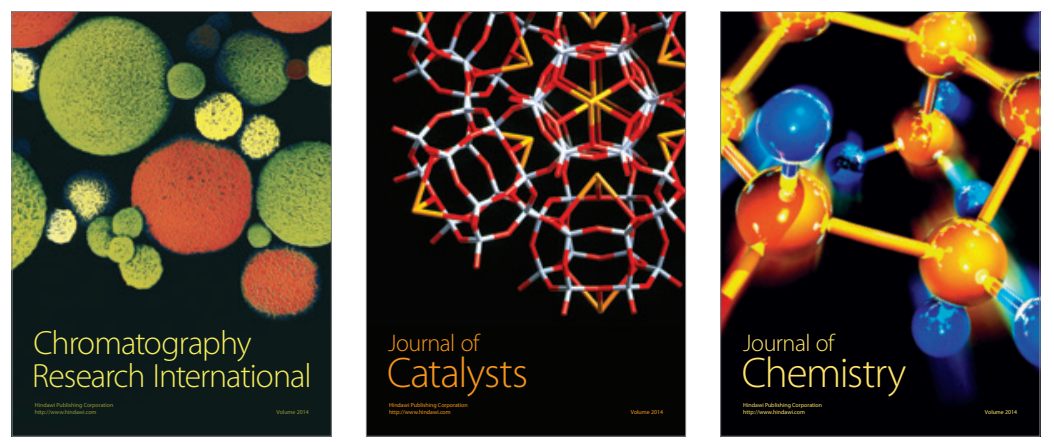
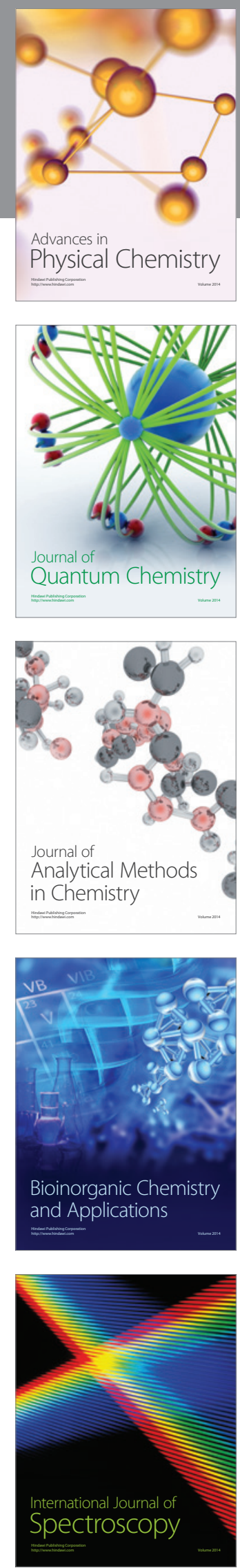